\title{
Review: professional or social support reduce postpartum depression
}

Ray KL, Hodnett ED. Caregiver support for postpartum depression. In: The Cochrane Library; issue 1. Oxford: Update Software, 1998.

\section{Question}

In women who have postpartum depression, are professional or social support programmes effective?

\section{Data sources}

Studies were identified using the Cochrane Pregnancy and Childbirth Group's Specialised Register of Controlled Trials; trials in this register were identified by searching Medline (from 1966), hand searching journals and conference proceedings, and contacting obstetricians and paediatricians in 18 countries.

\section{Study selection}

Studies were selected if they compared usual postpartum care for depressed mothers with additional professional or social support (or both), included women who had live infants and were clinically depressed during the first 6 months postpartum, used randomised or quasirandomised methods to allocate women to treatment groups, and the methodological quality was acceptable with no systematic error in the randomisation or follow up of patients. Studies which used pharmacological treatment were included. Studies on the prevention of postpartum depression were excluded.

\section{Data extraction}

Data were extracted on the methodological quality of the studies, the characteristics of the participants and interventions, and number of women who were considered to be depressed at 25 weeks postpartum. Unpublished data were obtained from the author of 1 study.

\section{Main results}

Meta-analytical techniques were used to pool the data. 2 randomised controlled trials in the UK involving 142 patients met the inclusion criteria; 111 patients completed the trials. 1 study $(\mathrm{n}=61)$ compared fluoxetine plus 1 or 6 counselling sessions that involved cognitive behavioural techniques with placebo plus 1 or 6 counselling sessions. 1 study $(n=50)$ compared weekly visits after 12 weeks postpartum by health visitors who were trained in non-directive counselling with usual postpartum care for depressed mothers. Fewer patients who received extra support had depression at 25 weeks postpartum compared with patients who received usual care $\{\mathrm{p}=0.002\}^{*}$ (table).

\section{Conclusion}

In 2 small trials, women who receive extra support were less likely to suffer continuing postpartum depression than patients who receive usual postpartum care for depressed mothers.

*p value calculated from data in article.

Professional or social support $v$ usual care at 25 weeks in women with postpartum depressiont

\begin{tabular}{llllll}
\hline & & \multicolumn{2}{l}{ Weighted event rates } & & \\
\cline { 3 - 4 } Outcome & \multirow{2}{*}{ No of studies } & Support & Usual care & RRR (95\% CI) & NNT (CI) \\
\hline Depression & 2 & $32 \%$ & $60 \%$ & $47 \%(18$ to 66$)$ & $4(3$ to 10)
\end{tabular}

$\dagger$ Abbreviations defined in glossary; RRR, NNT, and CI calculated from data in article.

Source of funding: no external funding.

For correspondence: Ms K L Ray, 19 Richdale Court, Etobicoke, Ontario M9B 5Y8, Canada.Fax +1 4162345391

\section{Commentary}

This useful systematic review by Ray and Hodnett shows the value of human support for women suffering from nonpsychotic postnatal depression. The Appleby study in Manchester, UK compared 6 counselling sessions with a psychologist over 11 weeks with a single 1 hour session with the same psychologist. ${ }^{1}$ Six sessions were better than 1, although most of the improvement occurred by 4 weeks (after just 3 sessions). The Holden study in Scotland compared 8 weekly sessions with a health visitor with usual care. ${ }^{2}$ Both studies showed that the number of women who were still depressed at 12 weeks was halved in the groups receiving extra support.

This review has limitations. It is confined to human support as a treatment. The Appleby study also compared fluoxetine with placebo and showed the drug to be as effective as the extra counselling sessions.
It is a pity that the review did not compare all treatments for postnatal depression. I sympathise with Ray and Hodnett: most randomised controlled trials are of pharmacological treatments and Ellen Hodnett, in particular, deserves recognition for bringing the value of human support in maternity care to our attention. None the less, some women might prefer pills to people in their course of treatment and the privacy of pharmacology to the presence of a psychologist. It was surprising that the effects of fluoxetine and counselling were not found, at least in Appleby's study, to be additive. Women should be offered a choice between pills or people in their course of treatment. Breast feeding women in particular may prefer to avoid drugs. Given the cost of healthcare salaries, I suspect many purchasers may not offer women such choice in the same way that electronic fetal monitors have replaced midwives in labour with possible deleterious results.

This review limits itself to treatment. Postnatal depression is a common condition found in different cultures throughout the world, ${ }^{3}$ and it probably results from an interaction of genetic, hormonal, and social factors. Preventing depression would be a major advance. Helping health carers to recognise postnatal depression would be an important step. This review provides evidence for 1 form of treatment.

Gavin Young, MA, FRCGP, DRCOG Temple Sowerby Surgery Penrith, UK

1 Appleby L, Warner R, Whitton A, et al. BMJ 1997;314:932-6.

2 Holden JM, Sagovsky R, Cox JL. BMJ 1989;298: 223-6.

3 Cox JL. Soc Psychiatry 1983;18:25-8. 